\title{
A CASE OF CATATONIA RELATED TO INFECTIVE ENDOCARDITIS DURING PREGNANCY
}

\author{
Ayşegül Şahin Ekici, Şengül Şahin, Bahadir Demir, Gülçin Elboğa \& Abdurrahman Altindağ \\ Department of Psychiatry Gaziantep Univercity Faculty of Medicine, Gaziantep, Turkey
}

received: 4.1.2021;

revised: 5.3.2021;

accepted: 17.3 .2021

\section{INTRODUCTION}

Catatonia is commonly related to schizophrenia; it is now known that catatonic symptoms are nonspecific and may be observed in other mental disorders and neurological conditions. (Walther et al. 2019)

It may also be seen in many medical disorders including infections (such as encephalitis), autoimmune disorders, meningitis, alcohol withdrawal, benzodiazepine withdrawal, cerebrovascular diseases, neoplasms, head injury, and some metabolic conditions.(Pot \& Lejoyeux 2015)

In this study, we reported a rare presentation of catatonia, the catatonia has been induced by Meticillin Resistant Staplylococcus Aureus (MRSA), in a pregnant woman, 28 years aged, who has not been diagnosed with any other psychiatric disease before. Cases of catatonia caused by infection are very rare and no other cases with induced by Stapylococcus Aureus have been reported according to our knowledge. We reported the first case of catatonia by may related to septicemia due to MRSA.

\section{CASE PRESENTATION}

A 28-year-old woman was presented by her family to our psychiatry outpatient clinic with complaints of reduced fluid and food intake, insomnia, and limited communication. We learned that at the initial stage, she displayed excessive mobility and suffered from insomnia for four days because she kept thinking that her relatives will harm her. Although her speech was disorganized, she had been speaking to herself, and she refused to go to the toilet or take a bath. It was also reported that she would lie down on the floor at inappropriate times, a behavior that lasted for three weeks.

There was no history of psychiatric illness or drug addiction in her anamnesis and those of her family. An initial assessment revealed that Bush-Francis Catatonia Rating Scale score of 11. Her neurological examination was unremarkable. 24-week pregnancy was detected with a healthy fetus. Her mental examination showed psychomotor excitation, negativism, reduced self-care, mutism, and a withdrawal of food and fluid intake. She only communicated with her older brother using a few words and had no verbal interaction with the physician.
We administered a daily dosage of olanzapine $20 \mathrm{mg}$ for the psychomotor excitation and insomnia. After preparing her for the procedure, we planned and performed an electroconvulsive therapy (ECT) session. Serum CRP (C-reactive protein) level was $29.56 \mathrm{mg} / 1$ (mildly high), the patient's condition worsened with a fever of $39.2^{\circ} \mathrm{C}$. ECT sessions were discontinued and she was transferred to the Infectious Diseases Department.

A transesophageal echocardiography was conducted, and the findings revealed an infective endocarditis, evidenced by a fever, tachycardia, and persistent CRP levels $(259.85 \mathrm{mg} / \mathrm{l})$. The echocardiography results also showed mobile vegetations in the anterior and posterior leaflets of the mitral valve, and a growth of methicillinresistant Staphylococcus aureus (MRSA) was observed in the blood culture.

It had been performed a termination of pregnancy with cesarean section; this was followed by the mitral valve replacement surgery. After intensive care, psychiatric evaluation revealed a decrease in excitation and a partial improvement in her refusal to eat; however, she displayed selective mutism. As a result, we recommended a daily administration of lorazepam $7.5 \mathrm{mg}$ during hospitalization. At discharge, the patient had low, disorganized speech and her Bush-Francis Catatonia Rating Scale score was 4. She was discharged from the infectious diseases' inpatient clinic after two months of infective endocarditis treatment. She did not come back to follow-up again.

\section{DISCUSSION}

The purpose of this report is to present the differential diagnosis of a pregnant patient presenting with mutism and catatonic excitation; the relationship between infective endocarditis and the formation of an infection-induced cranial abscess; and the importance of organic causes in the differential diagnosis of catatonia.

A study has shown that $20 \%$ of catatonia cases are associated with the occurrence of general diseases (Oldham 2018). A systematic review revealed that $25 \%$ of 124 infection-related catatonia cases found in the literature were caused by a systemic bacterial infection; Salmonella typhi was found in 29 cases, Coxiella burnetii was found in one, and no bacterial infection was detected in the last case (Walther et al. 2019). 
Antimicrobial therapy, in some cases, has shown promising results in treating infection-induced catatonia (Pfister et al. 1993); however, other cases require treatment with benzodiazepines or ECT.

There is a strong possibility that pathophysiological mechanisms are involved in cases of infection-induced catatonia. It is believed that the microorganisms, which are the sources of infection, have direct neurotoxic effects that can lead to a psychological reaction or an acute phase response. In clinical situations, such as meningoencephalitis where the central nervous system is affected, the damage has been shown to be associated with an immunological response (Waisman et al. 2015).

Increasing evidence in many neurological disorders show that inflammatory molecules released by Astrocytes and microglia mediate neurotoxicity. Grampositive bacterial cell walls (lipoteichoic acid) activate glial cells and secrete proinflammatory cytokines (TNF$\alpha$, IL-1) and other mediators (Nitricoxide, superoxide, peroxynitrite) that can cause oxidative stress, activation of caspase, mitochondrial dysfunction, and the eventual death of neurons (Kinsner et al. 2005).

An experimental study on rodents showed that brain infections caused by Staphylococcus aureus increased extracellular GABA ( $\gamma$-aminobutyric acid), glutamate, and aspartate levels, which indicate the degree of tissue damage. (Hassel et al. 2014)

\section{CONCLUSION}

We believe that the catatonia presented in this case may be related to proinflammatory cytokines, inflammation, or neurotoxicity brought about by toxins from Staphylococcus aureus bacteremia. The clinical symptoms of the patient appeared to occur after MRSA septicemia; hence it is thought that her catatonic condition was triggered by the septicemia. The etiology of catatonia has not been fully elucidated; however, it is believed that catatonia is caused by a complex mechanism influenced by many different pathophysiological pathways. This case presents another clue to the etiology of catatonia.
Acknowledgements: None.

\section{Conflict of interest: None to declare.}

\section{Contribution of individual authors:}

Ayşegül Şahin Ekici: research idea, study design, manuscript writing, literature search.

Şengül Şahin: manuscript revising, study design, literature search.

Bahadır Demir: study design, literature search.

Gülçin Elboğa: study design, case supervision.

Abdurrahman Altındağ: manuscript revising, case supervision.

\section{References}

1. Hassel B, Dahlberg D, Mariussen E, Goverud IL, Antal EA, Tonjum $T \&$ \& Maehlen J: Brain infection with Staphylococcus aureus leads to high extracellular levels of glutamate, aspartate, gamma-aminobutyric acid, and zinc. J Neurosci Res 2014; 92:1792-1800

2. Kinsner A, Pilotto V, Deininger S, Brown GC, Coecke S, Hartung $T \&$ Bal-Price A: Inflammatory neurodegeneration induced by lipoteichoic acid from Staphylococcus aureus is mediated by glia activation, nitrosative and oxidative stress, and caspase activation. Journal of neurochemistry 2005; 95:1132-1143

3. Oldham MA: The probability that catatonia in the hospital has a medical cause and the relative proportions of its causes: a systematic review. Psychosomatics 2018; 59:333-340

4. Pfister H-W, Preac-Mursic V, Wilske B, Rieder G, Förderreuther $S$, Schmidt $S$ \& Kapfhammer H-P: Catatonic syndrome in acute severe encephalitis due to Borrelia burgdorferi infection. Neurology 1993; 43:433-433

5. Pot A-L \& Lejoyeux M: La catatonie. L'Encéphale 2015; 41:274-279

6. Waisman A, Liblau $R S \&$ Becher B: Innate and adaptive immune responses in the CNS. The Lancet Neurology 2015; 14:945-955

7. Walther S, Stegmayer K, Wilson JE \& Heckers S: Structure and neural mechanisms of catatonia. The Lancet Psychiatry 2019; 6:610-619

Correspondence:

Research Assist. Ayşegül Şahin Ekici, MD

Department of Psychiatry Gaziantep, Univercity Faculty of Medicine

Gaziantep, Turkey

E-mail:asahinekici@gmail.com 